\title{
Impact of heterogeneity-corrected dose calculation using a grid-based Boltzmann solver on breast and cervix cancer brachytherapy
}

\author{
Julia Hofbaver, MSc',2, Prof. Christian Kirisits, DSc',3, Alexandra Resch, MD², Yingjie Xu, MSc', Alina Sturdza, MD², \\ Prof. Richard Pötter, MD 2,3 , Nicole Nesvacil, DSc ${ }^{2,3}$ \\ Institut für Krankenhausphysik, Krankenhaus Hietzing, Vienna, Austria, ${ }^{2}$ Department of Radiotherapy, Comprehensive Cancer Center, \\ Medical University of Vienna, Austria, ${ }^{3}$ Christian Doppler Laboratory for Medical Radiation Research for Radiation Oncology. \\ Medical University of Vienna, Austria, ${ }^{4}$ Department of Radiation Oncology, Chinese Academy of Medical Sciences, Beijing, China
}

\begin{abstract}
Purpose: To analyze the impact of heterogeneity-corrected dose calculation on dosimetric quality parameters in gynecological and breast brachytherapy using Acuros, a grid-based Boltzmann equation solver (GBBS), and to evaluate the shielding effects of different cervix brachytherapy applicators.

Material and methods: Calculations with TG-43 and Acuros were based on computed tomography (CT) retrospectively, for 10 cases of accelerated partial breast irradiation and 9 cervix cancer cases treated with tandem-ring applicators. Phantom CT-scans of different applicators (plastic and titanium) were acquired. For breast cases the $V_{20 G y a \beta 3}$ to lung, the $\mathrm{D}_{0.1 \mathrm{~cm}^{3},} \mathrm{D}_{1 \mathrm{~cm}^{3}}, \mathrm{D}_{2 \mathrm{~cm}^{3}}$ to rib, the $\mathrm{D}_{0.1 \mathrm{~cm}^{3}}, \mathrm{D}_{1 \mathrm{~cm}^{3}}, \mathrm{D}_{10 \mathrm{~cm}^{3}}$ to skin, and $\mathrm{D}_{\max }$ for all structures were reported. For cervix cases, the $\mathrm{D}_{0.1 \mathrm{~cm}^{3}}, \mathrm{D}_{2 \mathrm{~cm}^{3}}$ to bladder, rectum and sigmoid, and the $\mathrm{D}_{50}, \mathrm{D}_{90}, \mathrm{D}_{98}, \mathrm{~V}_{100}$ for the $\mathrm{CTV}_{\mathrm{HR}}$ were reported. For the phantom study, surrogates for target and organ at risk were created for a similar dose volume histogram (DVH) analysis. Absorbed dose and equivalent dose to 2 Gy fractionation (EQD2) were used for comparison.

Results: Calculations with TG-43 overestimated the dose for all dosimetric indices investigated. For breast, a decrease of $\sim 8 \%$ was found for $\mathrm{D}_{10 \mathrm{~cm}^{3}}$ to the skin and $5 \%$ for $\mathrm{D}_{2 \mathrm{~cm}^{3}}$ to rib, resulting in a difference $\sim-1.5$ Gy EQD2 for overall treatment. Smaller effects were found for cervix cases with the plastic applicator, with up to $-2 \%$ (-0.2 Gy EQD2) per fraction for organs at risk and $-0.5 \%$ (-0.3 Gy EQD2) per fraction for $\mathrm{CTV}_{\mathrm{HR}}$. The shielding effect of the titanium applicator resulted in a decrease of $2 \%$ for $\mathrm{D}_{2 \mathrm{~cm}^{3}}$ to the organ at risk versus $0.7 \%$ for plastic.

Conclusions: Lower doses were reported when calculating with Acuros compared to TG-43. Differences in dose parameters were larger in breast cases. A lower impact on clinical dose parameters was found for the cervix cases. Applicator material causes systematic shielding effects that can be taken into account.
\end{abstract}

Key words: Acuros, breast, brachytherapy, cervix, dose calculation.

\section{Purpose}

Dose calculation is essential for prescribing, recording, and reporting dose values in brachytherapy. An accurate dose assessment is the basis to optimize treatment plans for meeting the defined planning aims and for establishing dose-response curves to predict tumor control and morbidity. The current state of the art to calculate brachytherapy absorbed dose is the American Association of Physicists in Medicine (AAPM) Task Group No. 43 (TG-43) algorithm [1,2]. The formalism is based on the dose rate constant, geometry and radial dose functions, as well as anisotropy factor. Algorithms based on the TG-43 formalism utilize dose rate distributions pre-cal- culated in a standard, homogeneous water geometry. It neglects the specific variations in tissue composition and applicator material. Due to the different energy ranges between low energy photon sources, high energy photon sources and external beam radiation therapy (EBRT), the integration of tissue composition has been of lower priority compared to external beam therapy where state of the art dose calculation is based on the Hounsfield units of the irradiated tissue. However, particularly when including high $\mathrm{Z}$ materials for applicators and missing backscatter when treating close to the body surface uncertainties in dose reporting became obvious $[3,4,5,6]$. There is an increasing interest in development of more accurate dose calculation algorithms and
Address for correspondence: Nicole Nesvacil, DSc, Department of Radiotherapy, Medical University Vienna/AKH Vienna Währinger Gürtel 18-20, A-1090 Vienna, Austria, phone: +43140400 2692, fax: +43 140400 2693, $\bowtie$ e-mail: nicole.nesvacil@meduniwien.ac.at
Received: 25.11.2015

Accepted: 1.04 .2016

Published: 27.04.2016 
software solutions $[3,7,8]$. Recently, also the vendors of treatment planning software have included such algorithms in their systems. This study analyzes the impact of improved dosimetry by taking into account material inhomogeneities and patient specific scatter conditions, based on a commercially available dose planning software for two different clinical sites: breast and cervix cancer brachytherapy. The aim is to compare dose reporting based on TG-43 and a grid-based Boltzmann solver (GBBS), and to evaluate the clinical relevance of differences between these dose calculation methods.

\section{Material and methods}

\section{General}

Defined dose volume histogram (DVH) parameters from computed tomography $(\mathrm{CT})$-based treatment plans of randomly selected patients were analyzed using treatment planning system BrachyVision v10.0 (Varian Medical Systems, Palo Alto, CA, USA). Plans were created based both on TG-43 formalism and using the GBBS Acuros v1.4.0 algorithm $[9,10]$, and dose was reported as kerma-to-water in medium. Two cohorts of patients, for breast and cervix cancer brachytherapy were examined and an additional phantom study was performed for gynecological applicators. Simulated 3D image-based treatment plans were based on CT scans acquired with a Somatom Plus scanner (Siemens, Erlangen, Germany), and a slice thickness of $2 \mathrm{~mm}$ for breast and $4 \mathrm{~mm}$ for cervix brachytherapy plans, respectively. Absorbed dose values were compared, as well as clinically relevant equieffective doses using the EQD2 concept [11]. Throughout the study, an $\alpha / \beta$ ratio of 3 Gy was used for organs at risk and $10 \mathrm{~Gy}$ for target volumes with 1.5 hours for half-time of repair.

\section{Breast cases}

Ten cases of accelerated partial breast irradiation (APBI) with flexible plastic implants were analyzed. Dwell paths were digitized manually. Ipsilateral lung and the most exposed rib were contoured as regions of interest (Figure 1). A help structure for evaluating skin dose was delineated outside the skin surface as described in [12], the thickness of this structure being $4 \mathrm{~cm}$ from surface. Reported DVH-parameters were $\mathrm{V}_{20 \mathrm{Gya} 33}$ for lung, $\mathrm{D}_{0.1 \mathrm{cc}}$ $D_{1 c c}$ and $D_{2 c c}$ for rib, and $D_{0.1 c c}, D_{1 c c}$ and $D_{10 c c}$ for the skin help structure. Also for all structures $\mathrm{D}_{\max }$ was reported. These values were calculated based on the TG- 43 and Acuros algorithms and compared to each other. All treatment plans were generated based on our clinical protocol for a pulsed-dose-rate (PDR) schedule of 63 pulses and a total planning aim dose of $50.4 \mathrm{~Gy}$. The total treatment dose was calculated using the linear quadratic model.

\section{Cervix cases}

Also cases of 9 cervix cancer patients treated with plastic tandem-ring applicators (Nucletron, Veenendaal, The Netherlands) were scanned. For brachytherapy treatment planning, an HDR schedule consisting of four brachytherapy fractions was assumed. Five out of nine patients were treated with a combined intracavitary/ interstitial technique with 3 to 10 additional needles. Applicator source paths were digitized manually. Highrisk clinical target volume $\left(\mathrm{CTV}_{\mathrm{HR}}\right)$ as well as organs at risk (OAR) such as bladder, rectum, and sigmoid were contoured as regions of interest (Figure 1). The reported DVH parameters were $\mathrm{D}_{50}, \mathrm{D}_{90}, \mathrm{D}_{98}$, and $\mathrm{V}_{100}$ for the $C T V_{H R}$ and $D_{0.1 \mathrm{~cm}^{3}}$ and $\mathrm{D}_{2 \mathrm{~cm}^{3}}$ for the OAR, respectively. For Acuros, no additional density information than the CT data was used, as the system did not provide the fitting applicator model used in these cases.
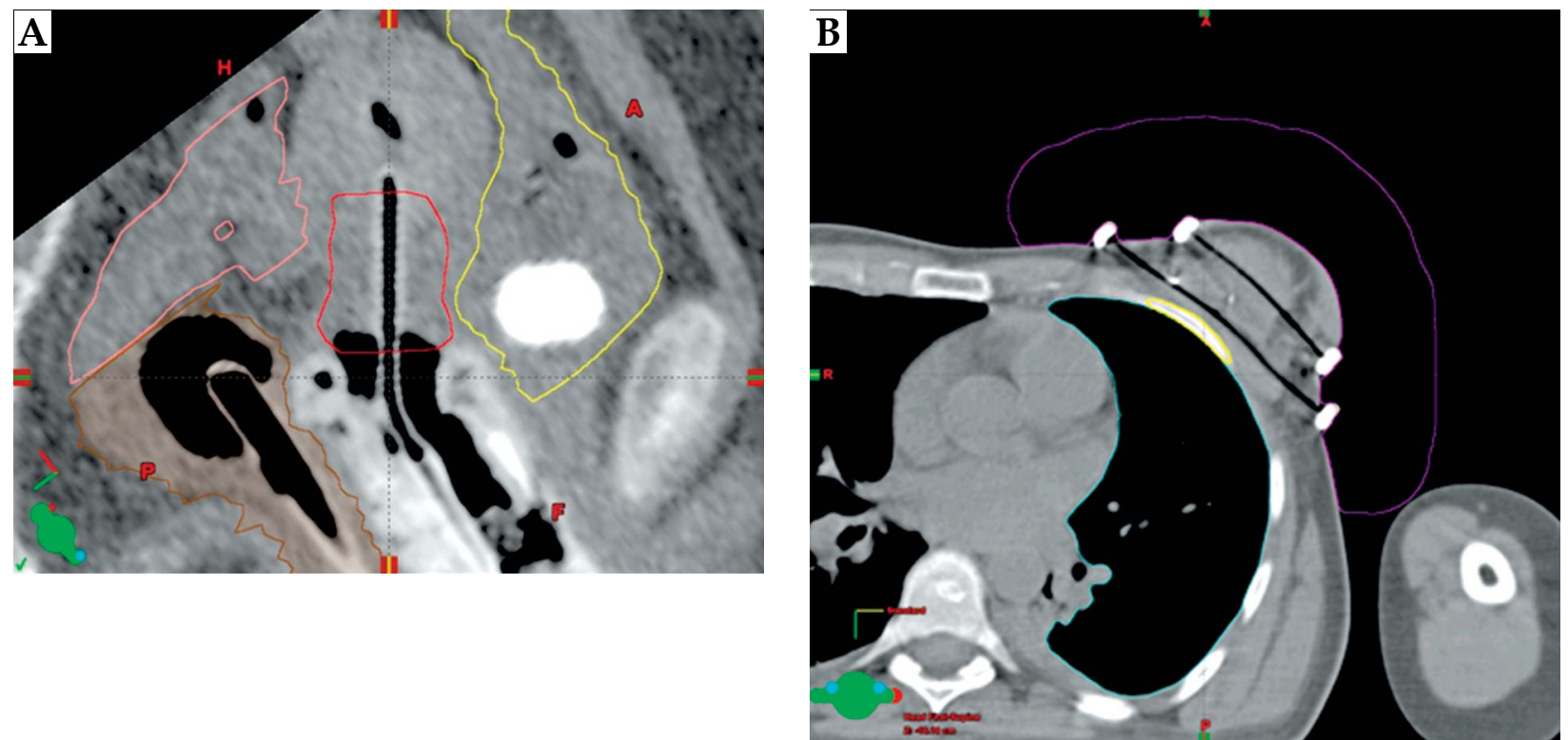

Fig. 1. Computed tomography slice showing target and organs at risk delineation. A) Clinical cervix case, in sagittally reconstructed applicator view, with high-risk clinical target volume $\left(\mathrm{CTV}_{\mathrm{HR}}\right)$, rectum, bladder, and sigmoid delineated. B) Transversal cut for a breast case, with skin surrogate, rib, and lung delineation 
Treatment plans were generated based on a typical clinical protocol for EBRT (45 Gy) and high-dose-rate (HDR) brachytherapy delivered in 4 fractions. The planning aim was to achieve a $\mathrm{D}_{90}$ for $\mathrm{CTV}_{\mathrm{HR}} \geq 7$ Gy per fraction, which results in a total treatment $\mathrm{D}_{90} \geq 84$ Gy EQD2, based on the linear quadratic model using $\alpha / \beta=10 \mathrm{~Gy}$. For OARs, the planning aims were to achieve a total $\mathrm{D}_{2 \mathrm{~cm}^{3}}$ $\leq 90,70$, and 75 Gy EQD2 using $\alpha / \beta=3 \mathrm{~Gy}$, for bladder rectum, and sigmoid, respectively.

\section{Phantom study}

In addition, a phantom study was executed to investigate the impact of applicator material only. Tandem-ring applicators built of titanium (Varian Medical Systems), as well as of the clinically used plastic tandem-ring applicators as mentioned above were CT-scanned in a water phantom. The plastic applicator was scanned twice without and with additional titanium needles inserted.

For the phantom plans, the applicator model of the titanium applicator was used from the library providing matching source path, as well as corresponding vendor-provided density information. Heterogeneity corrected dose calculation for the plastic applicator was based on CT-scan information only. Since both types of applicators were of comparable physical dimensions, the same dwell path was used for both in order to break down comparison to the applicator material. In this phantom study, a region near the applicator surface was created as a surrogate for an OAR, as well as a region around the applicator itself as surrogate for a target volume. Cor- responding dosimetric quality parameters were investigated as $D_{50}, D_{90}, D_{98}$, and $V_{100}$ for the target surrogate and $\mathrm{D}_{0.1 \mathrm{~cm}^{3}}$ and $\mathrm{D}_{2 \mathrm{~cm}^{3}}$ for the OAR surrogate, respectively.

\section{Library applicator analysis for different metals}

In order to test the difference between different types of metal applicators, 5 patient plans based on magnetic resonance imaging (MRI) were calculated using titanium and steel applicator model from the treatment planning system's applicator library. All plans were standard plans with no optimization and a prescribed dose of 7 Gy to point A. All plans were calculated with or without heterogeneity correction for the applicator material and differences between TG-43, and Acuros were reported for the $\mathrm{CTV}_{\mathrm{HR}} \mathrm{D}_{90}, \mathrm{D}_{98}$ and $\mathrm{D}_{50}$, and the rectum $\mathrm{D}_{0.1 \mathrm{~cm}^{3}}$ and $\mathrm{D}_{2 \mathrm{~cm}^{3}}$.

\section{Results}

\section{Breast cases}

A summary of the simulated treatment plans for the 10 breast cases is given in Table 1 . In general, TG- 43 calculated higher dose for breast cases compared to the Acuros dose calculation. All structures show an overestimation of dose when calculated by TG-43, because this algorithm is based on calculation in water. Lung tissue as well as medium outside the patient's surface do not provide the same amount of back scatter [13], which leads to lower doses calculated by the heterogeneity-corrected algorithm. Obviously, this effect results in a greater difference for skin surrogate of up to $-8 \%$ for the $\mathrm{D}_{10 \mathrm{~cm}^{3}}$, meaning

Table 1. Summary of dosimetric parameters calculated by TG-43 algorithm and GBBS Acuros in absorbed dose, as well as EQD2 showing results for organs at risk (skin surrogate, most exposed rib and lung) mean values \pm SD for overall treatment (63 pulses) of breast cases $(n=10)$

\begin{tabular}{|c|c|c|c|c|c|c|c|}
\hline & & \multicolumn{4}{|c|}{ Absorbed dose } & \multirow{2}{*}{\multicolumn{2}{|c|}{$\frac{\text { EQD2 }(\alpha / \beta=3 \text { Gy) }}{\text { Difference (Ac-TG-43) }}$}} \\
\hline & & \multirow{2}{*}{$\begin{array}{c}\text { TG-43 } \\
\text { Mean } \pm \text { SD } \\
(\mathrm{Gy})\end{array}$} & \multirow{2}{*}{$\begin{array}{c}\text { Acuros } \\
\text { Mean } \pm \text { SD } \\
(G y)\end{array}$} & \multicolumn{2}{|c|}{ Difference (Ac-TG-43) } & & \\
\hline & & & & $\begin{array}{c}\text { Mean } \pm \text { SD } \\
(\%) \\
\end{array}$ & $\begin{array}{c}\text { Range } \\
(\%)\end{array}$ & $\begin{array}{c}\text { Mean } \pm \text { SD } \\
\left(G y_{\text {iso }}\right)\end{array}$ & $\begin{array}{l}\text { Range } \\
\left(G y_{\text {iso }}\right)\end{array}$ \\
\hline \multirow[t]{4}{*}{ Skin } & $D_{\max }$ & $29.24 \pm 6.26$ & $28.07 \pm 6.35$ & $-4.18 \pm 1.45$ & {$[-5.84 ;-1.60]$} & $-1.60 \pm 0.53$ & {$[-2.51 ;-1.03]$} \\
\hline & $\mathrm{D}_{0.1 \mathrm{~cm}^{3}}$ & $26.26 \pm 5.19$ & $24.84 \pm 5.32$ & $-5.65 \pm 1.64$ & {$[-8.05 ;-2.30]$} & $-1.81 \pm 0.38$ & {$[-2.34 ;-1.29]$} \\
\hline & $\mathrm{D}_{1 \mathrm{~cm} \mathrm{c}^{3}}$ & $23.01 \pm 3.66$ & $21.52 \pm 3.70$ & $-6.65 \pm 1.46$ & {$[-8.70 ;-3.31]$} & $-1.79 \pm 0.34$ & {$[-2.28 ;-1.18]$} \\
\hline & $\mathrm{D}_{10 \mathrm{~cm}^{3}}$ & $17.80 \pm 2.34$ & $16.34 \pm 2.22$ & $-8.21 \pm 1.33$ & {$[-10.17 ;-5.46]$} & $-1.55 \pm 0.33$ & {$[-2.07 ;-0.97]$} \\
\hline \multirow[t]{4}{*}{ Rib } & $\mathrm{D}_{\max }$ & $41.59 \pm 17.78$ & $40.84 \pm 17.82$ & $-2.34 \pm 1.55$ & {$[-5.33 ;-0.58]$} & $-1.27 \pm 0.64$ & {$[-2.45 ;-0.64]$} \\
\hline & $\mathrm{D}_{0.1 \mathrm{~cm}^{3}}$ & $35.10 \pm 12.89$ & $34.19 \pm 12.76$ & $-2.96 \pm 1.27$ & {$[-5.50 ;-1.45]$} & $-1.45 \pm 0.62$ & {$[-2.47 ;-0.62]$} \\
\hline & $\mathrm{D}_{1 \mathrm{~cm} \mathrm{c}^{3}}$ & $28.05 \pm 9.39$ & $26.99 \pm 9.14$ & $-4.00 \pm 1.10$ & {$[-6.17 ;-2.73]$} & $-1.48 \pm 0.64$ & {$[-2.58 ;-0.60]$} \\
\hline & $\mathrm{D}_{2 \mathrm{~cm}}$ & $22.30 \pm 7.56$ & $21.25 \pm 7.33$ & $-4.92 \pm 1.13$ & {$[-6.61 ;-3.26]$} & $-1.28 \pm 0.54$ & {$[-2.23 ;-0.59]$} \\
\hline \multirow[t]{4}{*}{ Lung } & $\mathrm{D}_{\max }$ & $27.31 \pm 10.03$ & $26.14 \pm 9.75$ & $-4.92 \pm 1.35$ & {$[-6.82 ;-2.87]$} & $-1.62 \pm 0.69$ & {$[-2.79 ;-0.58]$} \\
\hline & $V_{\text {20GyEQD2 }}$ & \multicolumn{4}{|c|}{20 Gy volume in EQD2 } & & \\
\hline & & $(\%)$ & (\%) & $(\%)$ & $(\%)$ & & \\
\hline & & $0.69 \pm 0.45$ & $0.51 \pm 0.38$ & $-0.12 \pm 0.12$ & {$[-0.32 ; 0.00]$} & & \\
\hline
\end{tabular}

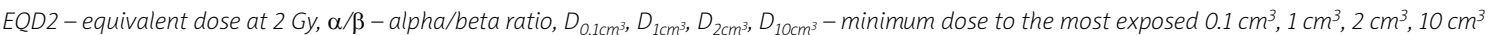


Table 2. Summary of dosimetric parameters calculated by TG-43 algorithm and GBBS Acuros in absorbed dose, as well as EQD2 showing results for target and organs at risk for cervix cases $(n=9)$. Mean values \pm SD per fraction are given for bladder, rectum, sigmoid, and CTV $_{\mathrm{HR}}$

\begin{tabular}{|c|c|c|c|c|c|c|c|}
\hline & & \multicolumn{4}{|c|}{ Absorbed dose } & \multirow{2}{*}{\multicolumn{2}{|c|}{$\frac{\text { EQD2 ( } \alpha / \beta=3 \text { resp. } 10 \text { Gy) }}{\text { Difference }(A c-T G-43)}$}} \\
\hline & & \multirow{2}{*}{$\begin{array}{c}\text { TG-43 } \\
\text { Mean } \pm \text { SD } \\
(\mathrm{Gy})\end{array}$} & \multirow{2}{*}{$\begin{array}{c}\text { Acuros } \\
\text { Mean } \pm \text { SD } \\
(G y) \\
\end{array}$} & \multicolumn{2}{|c|}{ Difference (Ac-TG-43) } & & \\
\hline & & & & $\begin{array}{c}\text { Mean } \pm \text { SD } \\
(\%) \\
\end{array}$ & $\begin{array}{c}\text { Range } \\
\text { (\%) }\end{array}$ & $\begin{array}{c}\text { Mean } \pm S D \\
\left(G y_{1 S 0}\right) \\
\end{array}$ & $\begin{array}{l}\text { Range } \\
\left(G y_{150}\right)\end{array}$ \\
\hline \multirow[t]{2}{*}{ Bladder } & $\mathrm{D}_{0.1 \mathrm{~cm}^{3}}$ & $5.56 \pm 1.12$ & $5.53 \pm 1.11$ & $-0.63 \pm 0.27$ & {$[-0.90 ; 0.00]$} & $-0.10 \pm 0.06$ & {$[-0.20 ; 0.00]$} \\
\hline & $\mathrm{D}_{2 \mathrm{~cm}^{3}}$ & $4.03 \pm 0.83$ & $4.00 \pm 0.82$ & $-0.87 \pm 0.25$ & {$[-1.41 ;-0.56]$} & $-0.08 \pm 0.04$ & {$[-0.17 ;-0.04]$} \\
\hline \multirow[t]{2}{*}{ Rectum } & $\mathrm{D}_{0.1 \mathrm{~cm}^{3}}$ & $3.12 \pm 0.67$ & $3.07 \pm 0.67$ & $-1.42 \pm 0.72$ & {$[-2.52 ;-0.62]$} & $-0.08 \pm 0.05$ & {$[-0.18 ;-0.03]$} \\
\hline & $\mathrm{D}_{2 \mathrm{~cm}^{3}}$ & $2.31 \pm 0.54$ & $2.26 \pm 0.53$ & $-2.14 \pm 0.63$ & {$[-3.27 ;-1.32]$} & $-0.08 \pm 0.03$ & {$[-0.12 ;-0.04]$} \\
\hline \multirow[t]{2}{*}{ Sigmoid } & $\mathrm{D}_{0.1 \mathrm{~cm}^{3}}$ & $5.19 \pm 1.13$ & $5.12 \pm 1.14$ & $-1.43 \pm 0.65$ & {$[-2.41 ;-0.34]$} & $-0.19 \pm 0.08$ & {$[-0.33 ;-0.06]$} \\
\hline & $\mathrm{D}_{2 \mathrm{~cm}^{3}}$ & $3.73 \pm 0.99$ & $3.67 \pm 0.99$ & $-1.69 \pm 0.81$ & {$[-2.97 ;-0.43]$} & $-0.12 \pm 0.05$ & {$[-0.22 ;-0.05]$} \\
\hline \multirow[t]{5}{*}{$\mathrm{CTV}_{\mathrm{HR}}$} & $\mathrm{D}_{50}$ & $11.08 \pm 1.25$ & $11.08 \pm 1.30$ & $-0.10 \pm 0.53$ & {$[-0.87 ; 0.68]$} & $-0.01 \pm 0.17$ & {$[-0.23 ; 0.28]$} \\
\hline & $\mathrm{D}_{90}$ & $7.61 \pm 0.59$ & $7.57 \pm 0.60$ & $-0.47 \pm 0.33$ & {$[-1.25 ;-0.14]$} & $-0.07 \pm 0.05$ & {$[-0.18 ;-0.02]$} \\
\hline & $\mathrm{D}_{98}$ & $6.38 \pm 0.49$ & $6.35 \pm 0.50$ & $-0.50 \pm 0.41$ & {$[-1.42 ; 0.00]$} & $-0.06 \pm 0.04$ & {$[-0.14 ; 0.00]$} \\
\hline & $V_{100}$ & [\%] & {$[\%]$} & [\%] & [\%] & & \\
\hline & & $98.89 \pm 0.95$ & $98.72 \pm 1.01$ & $-0.17 \pm 0.16$ & {$[-0.51 ; 0.00]$} & & \\
\hline
\end{tabular}

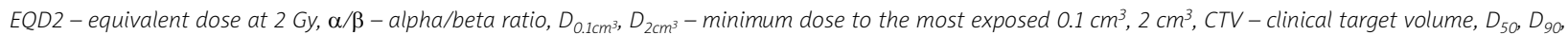
$D_{98}$ - the minimum dose to $50 \%, 90 \%, 98 \%$ of the CTV, $V_{100}$-volume of the anatomic volume receiving $100 \%$, of the prescribed dose

-1.5 Gy EQD2 for overall treatment of 63 pulses. Similar results can be found for the most exposed ribs where the mean $\mathrm{D}_{2 \mathrm{~cm}^{3}}$ was $-1.3 \mathrm{~Gy}$ in EQD2 or nearly $-5 \%$ in absorbed dose. Correspondingly, the dose difference of ipsilateral lung shows about the same percentage of -1.6 Gy EQD2 in the overall treatment. The mean equivalent volume of 20 Gy EQD2 with an $\alpha / \beta$ of 3 Gy is about $1.5 \mathrm{~cm}^{3}$ smaller on average for heterogeneity corrected calculation.

\section{Cervix cases}

An overview of the results for the 9 investigated cervix cases is given in Table 2. The comparison of dose calcula-

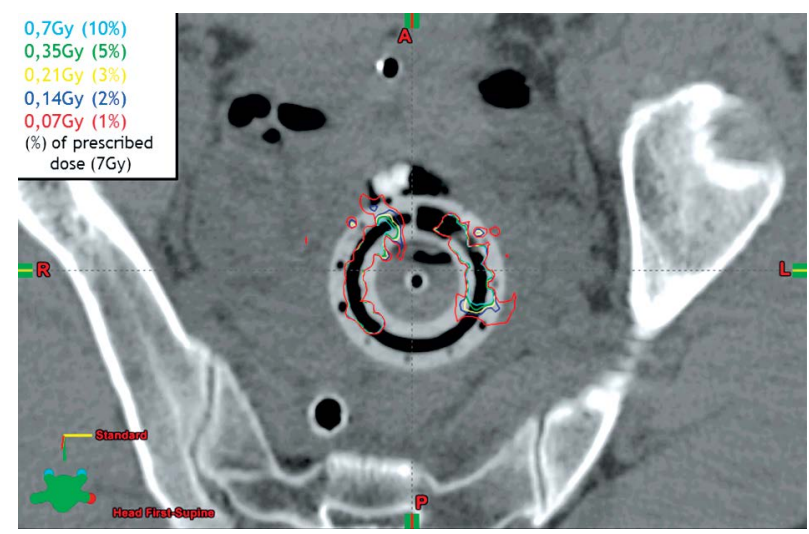

Fig. 2. Dose difference map for a plastic ring applicator. Relevant differences are limited to the vicinity of the applicator tion algorithms shows only a small impact on the cervix cases investigated when using a plastic applicator (Figure 2). Mean differences in absorbed dose for $\mathrm{D}_{0.1 \mathrm{~cm}^{3}}$ and $\mathrm{D}_{2 \mathrm{~cm}^{3}}$ ranged between approximately $-1 \%$ and $-2 \%$ for organs at risk resulting in negligible a difference of EQD2 less than 0.2 Gy per fraction, on average. For a total treatment of 4 fractions, this would result in a systematic difference of less than $1 \mathrm{~Gy}$.

Even less impact was found for the $\mathrm{CTV}_{\mathrm{HR}}$, as the differences for investigated clinical parameters ranged from $-0.1 \%$ to $-0.5 \%$ in dose, which was equivalent up to only 0.07 Gy EQD2 per fraction. When dividing the results between intracavitary only and additional needle cases, no significant difference could be observed for all parameter except $D_{50}$. For the $D_{50}$, the mean absorbed dose difference for intracavitary/interstitial cases was $-0.5 \%$ compared to $0.4 \%$ for plain intracavitary cases.

\section{Phantom study}

Regarding the phantom, the results showed shielding effects of applicator material (Table 3 ), $\mathrm{D}_{90}$ to the defined target surrogate was overestimated by TG- 43 vs. Acuros by more than $1 \%$ for the titanium applicator and $\sim 0.5 \%$ for plastic. Clinically, this would result in an overestimation of dose of less than 0.1 Gy EQD2 per fraction for plastic applicator, whereas for titanium we observed more than double the effect. For 4 fractions using a titanium applicator, this adds up to 0.8 Gy EQD2 difference. Due to the overall lower doses for OAR's, the relative difference on $\mathrm{D}_{2 \mathrm{~cm}^{3}}$ was around $2 \%$ for titanium and about $0.7 \%$ for plastic, respectively. Clinically, this results in an over- 
estimation of dose by TG-43 of less than 0.1 Gy EQD2 per fraction for plastic applicator and about twice the value, as seen in the outcomes for the target surrogate structure. For a simulated full treatment of 4 HDR fractions using a titanium applicator, this results in 0.8 Gy EQD2 less for Acuros-based values compared to TG-43.

\section{Applicator library study for metals}

For the test of different metal applicators based on applicator library models, 10 treatment plans were compared. The results are reported in Table 4. As expected, the difference between TG-43 and Acuros was larger for the steel applicator, both for the DVH parameters of the target, and of the rectum. For the rectum, $\mathrm{D}_{0.1 \mathrm{~cm}^{3}}$ and $\mathrm{D}_{2 \mathrm{~cm}^{3}}$ were found to be on average $\sim 3 \%$ larger when the applicator material was taken into account for dose calculation in the case of steel. This was about double the effect that was observed when the same cases were calculated with a titanium applicator model.

Table 3. Dose differences for phantom study split in comparison for titanium and plastic applicator for organ at risk and target surrogate. A negative difference means the value was higher for TG-43 than for Acuros-based treatment plans

\begin{tabular}{|c|c|c|c|c|c|c|c|c|}
\hline & & & \multicolumn{3}{|c|}{ Absorbed dose } & \multicolumn{3}{|c|}{ EQD2 ( $\alpha / \beta=3$ resp. 10 Gy) } \\
\hline & & & $\begin{array}{c}\text { TG-43 } \\
\text { (Gy) }\end{array}$ & $\begin{array}{c}\text { Acuros } \\
\text { (Gy) }\end{array}$ & $\begin{array}{l}\text { rel } \Delta \\
(\%) \\
\end{array}$ & $\begin{array}{l}\text { TG-43 } \\
\left(G y_{\text {ISO }}\right) \\
\end{array}$ & $\begin{array}{l}\text { Acuros } \\
\left(G y_{150}\right) \\
\end{array}$ & $\begin{array}{l}\text { rel } \Delta \\
(\%) \\
\end{array}$ \\
\hline \multirow[t]{4}{*}{ OAR } & $\mathrm{D}_{0.1 \mathrm{~cm}^{3}}$ & Titanium & 5.32 & 5.22 & -1.88 & 8.85 & 8.58 & -3.06 \\
\hline & & Plastic & 4.95 & 4.92 & -0.61 & 7.87 & 7.79 & -0.98 \\
\hline & $\mathrm{D}_{2 \mathrm{~cm}^{3}}$ & Titanium & 4.43 & 4.34 & -2.03 & 6.58 & 6.37 & -3.22 \\
\hline & & Plastic & 4.23 & 4.20 & -0.71 & 6.12 & 6.05 & -1.12 \\
\hline \multirow[t]{9}{*}{ Target } & $\mathrm{D}_{50}$ & Titanium & 11.43 & 11.28 & -1.24 & 20.40 & 20.02 & -1.89 \\
\hline & & Plastic & 11.25 & 11.24 & -0.02 & 19.91 & 19.90 & -0.04 \\
\hline & $\mathrm{D}_{90}$ & Titanium & 7.54 & 7.44 & -1.24 & 11.02 & 10.82 & -1.76 \\
\hline & & Plastic & 7.51 & 7.47 & -0.51 & 10.96 & 10.88 & -0.73 \\
\hline & $\mathrm{D}_{98}$ & Titanium & 6.79 & 6.71 & -1.11 & 9.49 & 9.35 & -1.56 \\
\hline & & Plastic & 6.77 & 6.77 & -0.05 & 9.47 & 9.46 & -0.07 \\
\hline & $V_{100}$ & & $(\%)$ & $(\%)$ & $(\%)$ & & & \\
\hline & & Titanium & 96.37 & 95.35 & -1.02 & & & \\
\hline & & Plastic & 96.14 & 95.75 & -0.39 & & & \\
\hline
\end{tabular}

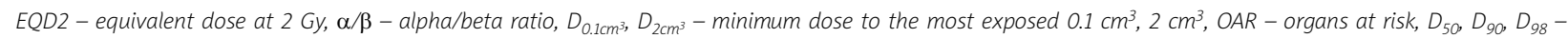
the minimum dose to $50 \%, 90 \%, 98 \%$ of the target, $V_{100}$ - volume of the anatomic volume receiving $100 \%$, of the prescribed dose

Table 4. Dose differences for library titanium and steel applicators for CTVHR and rectum, calculated on magnetic resonance imaging. A negative difference means the value was higher for TG-43 than for Acuros-based treatment plans

\begin{tabular}{|c|c|c|c|c|c|}
\hline \multicolumn{2}{|c|}{ Absorbed dose Ac-TG43 } & \multicolumn{2}{|c|}{ Titanium } & \multicolumn{2}{|c|}{ Steel } \\
\hline & $n=5$ & $\operatorname{rel} \Delta(\%)$ & SD (\%) & rel $\Delta(\%)$ & SD (\%) \\
\hline \multirow[t]{2}{*}{ Rectum } & $\mathrm{D}_{0.1 \mathrm{~cm}^{3}}$ & 1.35 & 0.81 & 2.70 & 0.92 \\
\hline & $\mathrm{D}_{2 \mathrm{~cm}} \mathrm{c}^{3}$ & 1.58 & 0.77 & 3.29 & 0.78 \\
\hline \multirow[t]{5}{*}{$C T V_{H R}$} & $D_{50}$ & 0.43 & 0.21 & 1.49 & 0.21 \\
\hline & $D_{90}$ & 0.58 & 0.12 & 1.61 & 0.21 \\
\hline & $D_{98}$ & 0.70 & 0.37 & 1.89 & 0.45 \\
\hline & $V_{100}$ & (\%) & $(\%)$ & $(\%)$ & $(\%)$ \\
\hline & & 0.22 & 0.23 & 0.68 & 0.67 \\
\hline
\end{tabular}

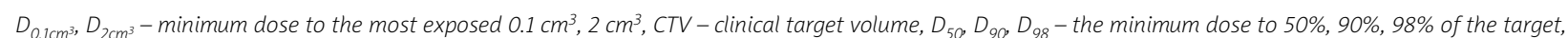
$V_{100}$-volume of the anatomic volume receiving $100 \%$, of the prescribed dose 


\section{Discussion}

The AAPM TG-43 algorithm is state of the art as it provides reliable dose distributions for clinical plans and its limitations are known. The results of this study indicate that use of heterogeneity correction shows effects on dose parameters, yet with no considerable clinical effect. Two main issues are found as expected. Whereas TG- 43 calculation tends to result in overestimation of dose where lack of backscatter plays a role in APBI, possible shielding effects of applicator material might show an impact in cervix cases.

For high-energy sources as ${ }^{192} \mathrm{Ir}$, the effect of missing back scatter due to the finite patient size has been described in detail $[3,4,5]$. Target volumes close to the skin or air filled cavities are mainly present in case of breast brachytherapy or head and neck brachytherapy [14]. Perturbations based on media different than water in case of high-energy sources have been mainly reported for shielded applicators and balloon catheters, filled with air or contrast medium. In this study, multi-catheter plastic implants are studied.

According to patient scatter, our study showed expected dosimetric uncertainties [6] in breast cases close to density gradients in tissue near lung and skins surface. The observed mean differences of the skin dose parameters based on different algorithms range from $4.2 \%$ for $D_{\text {max }}$ to $8.2 \%$ for $D_{10 \mathrm{~cm}^{3}}$. These ranges are in agreement with a $5-10 \%$ difference for skin point doses at different distances from the breast center, calculated with TG-43 and Monte Carlo simulations [4]. The difference of $\mathrm{D}_{\max }$ of lung and ribs has been previously reported for TG-43- and Acuros-based calculations of interstitial implants with metal catheters [15]. Reported results seem to indicate a $\sim 2 \%$ larger impact of metal catheters on the differences in $\mathrm{D}_{\max }$ in comparison to the present results for plastic catheters. A direct comparison of reported skin doses is unreliable, as in addition to different applicator materials also the definition of the skin structure for DVH evaluation was different in the present study. Zourari et al. reported $4 \%$ difference of $\mathrm{D}_{\max }$ for lung and rib when comparing TG-43 and a different commercially available model based dose calculation algorithm [16], which is in the range of the $\sim 2 \%$ and $\sim 5 \%$ differences we have observed for rib and lung for Acuros. As in the present study, they found the largest difference for the $D_{10 \mathrm{~cm}^{3}}$ of the skin $(6 \% \mathrm{vs} .8 \%$ in the present study).

Even though the effect of using an advanced dose calculation algorithm as such is systematic, its impact on dose parameters is location dependent. Therefore, individual planning in principle adds to more accuracy of dose-response for retrospective analysis and prospective treatment planning.

This effect could not be found in cervix cases. Effects due to rectum filling or packing causing low density or even areas filled with air had no major impact on dosimetric parameters analyzed. However, the phantom study showed an impact of applicator material when the titanium applicator seems to have more of a shielding effect compared to the plastic. Although this effect was small $(<2 \%)$, this might lead to a systematic shift of dose re- sponse curves [17]. Only in dose response modelling as it can be performed via large clinical trials (e.g. EMBRACE - http://www.embracestudy.dk [18]), it can be tested if a systematic offset in dose correlates better with clinical outcome.

Overall, the findings of our study are consistent with recent reports of DVH parameter deviations for target and OAR's, for Acuros-based dose calculations for cervix cancer brachytherapy with tandem/ovoid applicators [19] and Monte Carlo simulations [20]. In contrast to the findings reported by Mikell et al. [21], none of our cases showed differences between the dose calculation methods larger than $5 \%$. However, in the latter study, these large deviations were due to the influence of rectal contrast used for imaging. In addition, major deviations might be found when comparing point dose rather than volumes, since these might be less sensitive to intrinsic parameters of a grid-based calculation method. Especially in regions of dose gradients, spatial resolution might have a higher impact on differences in calculated dose too. Therefore, we think that for comparing these algorithms dosimetric parameters based on volumes should be favored over those based on singularities [21].

This study shows that calculation without heterogeneity correction may cause deviations of clinically used dosimetric parameters. However, this should not have an impact on common dose constraints as these refer to data retrieved from use of TG-43 algorithm. In line with the joint AAPM and GEC-ESTRO report, TG-186 dose calculation should be performed both ways.

In the present study, part of the applicator volume was included in the delineated target structure. The analysis of the $\mathrm{D}_{50}$, i.e. the minimum dose to $50 \%$ of the target volume, indicated that this high dose parameter is more sensitive to the influence of applicator volume inside the defined target volume. In this case, the effect of calculating DVH for volumes containing tissue and applicator material, versus the more correct calculation of DVH for the tissue volume only, might play a bigger role than heterogeneity correction.

\section{Conclusions}

The impact of heterogeneity corrected dose calculation using a grid-based Boltzmann solver on breast and cervix cancer brachytherapy was investigated with clinical treatment planning and phantom studies. For the studied situations of non-shielded applicators and a high-energy source, dose deviations are small. However, the known systematic overestimation of the skin dose in case of breast is confirmed, also taking into account biologically equieffective dose values. A small systematic dose effect was found when replacing intracavitary plastic applicators with titanium applicators, which is increasing when using steel applicators.

The observed effects for breast and cervix cases were not considered to be clinically relevant. However, advanced dose calculation tools such as Acuros can help to identify systematic effects and increase confidence in individual planning dosimetry and dose reporting in the future. 


\section{Acknowledgements}

This work was supported by Varian Medical Systems through a research grant.

\section{Disclosure}

The Department of Radiotherapy at the Medical University of Vienna receives financial and/or equipment support for research and educational purposes from Elekta $\mathrm{AB}$ and Varian Medical Systems Inc. C. Kirisits was a consultant to Elekta AB.

\section{References}

1. Rivard MJ, Coursey BM, DeWerd LA et al. Update of AAPM Task Group No. 43 Report: A revised AAPM protocol for brachytherapy dose calculations. Med Phys 2004; 31: 633-674.

2. Nath R, Anderson LL, Luxton G et al. Dosimetry of interstitial brachytherapy sources: recommendations of the AAPM Radiation Therapy Committee Task Group No. 43. American Association of Physicists in Medicine. Med Phys 1995; 22: 209-234

3. Lymperopoulou G, Papagiannis P, Angelopoulos A et al. A dosimetric comparison of $169 \mathrm{Yb}$ and 192Ir for HDR brachytherapy of the breast, accounting for the effect of finite patient dimensions and tissue inhomogeneities. Med Phys 2006; 33: 4583-4589.

4. Pantelis E, Papagiannis P, Karaiskos P et al. The effect of finite patient dimensions and tissue inhomogeneities on dosimetry planning of 192Ir HDR breast brachytherapy: a Monte Carlo dose verification study. Int J Radiat Oncol Biol Phys 2005; 61: 1596-1602.

5. Poon E, Verhaegen F. Development of a scatter correction technique and its application to HDR 192Ir multicatheter breast brachytherapy. Med Phys 2009; 36: 3703-3713.

6. Kirisits C, Rivard MJ, Baltas D et al. Review of clinical brachytherapy uncertainties: analysis guidelines of GEC-ESTRO and the AAPM. Radiother Oncol 2014; 110: 199-212.

7. Beaulieu L, Carlsson Tedgren A, Carrier JF et al. Report of the Task Group 186 on model-based dose calculation methods in brachytherapy beyond the TG-43 formalism: current status and recommendations for clinical implementation. Med Phys 2012; 39: 6208-6236.

8. Rivard MJ, Venselaar JL, Beaulieu L. The evolution of brachytherapy treatment planning. Med Phys 2009; 36: 2136-2153.

9. Petrokokkinos L, Zourari K, Pantelis E et al. Dosimetric accuracy of a deterministic radiation transport based 192Ir brachytherapy treatment planning system. Part II: Monte Carlo and experimental verification of a multiple source dwell position plan employing a shielded applicator. Med Phys 2011; 38: 1981-1992.

10. Zourari K, Pantelis E, Moutsatsos A et al. Dosimetric accuracy of a deterministic radiation transport based 192Ir brachytherapy treatment planning system. Part I: single sources and bounded homogeneous geometries. Med Phys 2010; 37: 649-661.

11. Bentzen SM, Dorr W, Gahbauer R et al. Bioeffect modeling and equieffective dose concepts in radiation oncology-terminology, quantities and units. Radiother Oncol 2012; 105: 266-268.

12. Berger D, Kauer-Dorner D, Seitz W et al. Concepts for critical organ dosimetry in three-dimensional image-based breast brachytherapy. Brachytherapy 2008; 7: 320-326.

13. Mangold CA, Rijnders A, Georg D et al. Quality control in interstitial brachytherapy of the breast using pulsed dose rate: treatment planning and dose delivery with an Ir-192 afterloading system. Radiother Oncol 2001; 58: 43-51.
14. Siebert FA, Wolf S, Kóvacs G. Head and neck (192)Ir HDRbrachytherapy dosimetry using a grid-based Boltzmann solver. J Contemp Brachytherapy 2013; 5: 232-235.

15. Sinnatamby M, Nagarajan V, Reddy KS et al. Dosimetric comparison of Acuros BV with AAPM TG43 dose calculation formalism in breast interstitial high-dose-rate brachytherapy with the use of metal catheters. I Contemp Brachytherapy 2015; 7: 273-279.

16. Zourari K, Major T, Herein A et al. A retrospective dosimetry comparison of TG43 and a commercially available MBDCA algorithm for an APBI brachytherapy patient cohort. Phys Med 2015; 31: 669-676.

17. Nesvacil N, Tanderup K, Kallehauge J et al. Impact of random dosimetric uncertainties on dose-response curves for HDR cervix cancer brachytherapy. Radiother Oncol 2013; 106, Suppl. 2: S141.

18. Tanderup K, Fokdal LU, Sturdza AE et al. Dose and Volume Response for Local Control in Locally Advanced Cervical Cancer Treated With EBRT Combined With MRI-Guided Adaptive Brachytherapy. Int J Radiat Oncol Biol Phys 2014; 90: S91-92.

19. Hyer DE, Sheybani A, Jacobson GM et al. The dosimetric impact of heterogeneity corrections in high-dose-rate ${ }^{192} \mathrm{Ir}$ brachytherapy for cervical cancer: Investigation of both conventional Point-A and volume-optimized plans. Brachytherapy 2012; 11: 515-520.

20. Al-Halabi H, Poon E, Portelance L et al. Comparison between Cone Beam CT Based Three-dimensional Planning and Monte Carlo dose Calculations in Intracavitary Brachytherapy for Cervical Cancer. Int J Radiat Oncol Biol Phys 2009; 75: S623.

21. Mikell JK, Klopp AH, Gonzalez GM et al. Impact of heterogeneity-based dose calculation using a deterministic grid-based Boltzmann equation solver for intracavitary brachytherapy. Int J Radiat Oncol Biol Phys 2012; 83: e417-422. 